\title{
CONSTRUCTION ASPECTS OF SYSTEM FOR WIRELESS LOW POWER TRANSFER ON MOVING PARTS OF ELECTRIC MACHINERY
}

The paper discuses important construction aspects of resonant wireless power transfer (WPT) system for moving parts of electric machinery. It focuses mainly on key electrical parameters of the systems resonant linkage and its simple and durable construction shape. All presented theoretical presumptions are consequently verified by detailed measurement.

Keywords: Wireless, power, transfer, resonant, coupling, efficiency.

\section{Introduction}

In the last few years there has been a growing interest in wireless power transfer systems. Considerable attention has been paid mainly to the possibilities in construction [1], [2] and [3] and the design of various systems structures. The mathematical description of operating characteristics and properties has been closely studied [4], [5], [6] and [7] by many researchers and it is therefore possible to qualitatively compare several variations of arrangement of relevant systems coils.

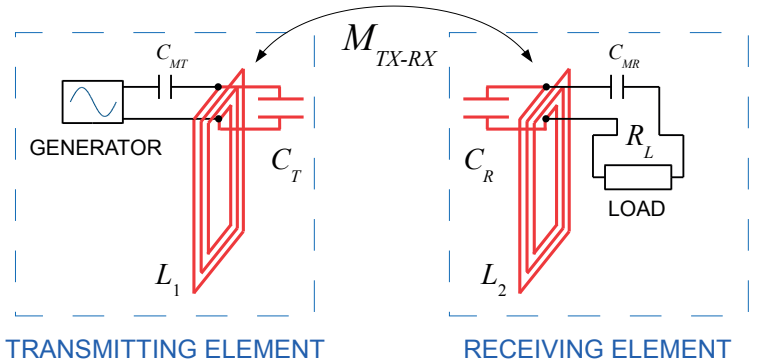

Fig. 1 The schematic diagram of system's construction

In this paper the two rectangular coils construction [4], [5] and [6] (see Fig. 1) of WPT system is studied. The operating frequency $13.56 \mathrm{MHz}$ is permitted by the scientific and industrial (ISM) band [7]. Based on the approach presented in [4], [5] and [6], the purpose of this paper is to study the impact on the efficiency of WPT within changing relations between chosen key elements of the structure. The analyzed modifications lie in various inductances of coils $L_{1}$ and $L_{2}$. Parameter $M_{T X-R X}$ represents the mutual inductance between respective coils. The capacitors $C_{M T}$ and $C_{M R}$ work as components of capacitive coupling matching the impedance of the system. This causes less decrease in value of the operational quality factor $Q$ of resonators $L_{1}$ and $L_{2}$ and prevents reflecting the power back to the source. Capacities $C_{T}$ and $C_{R}$ adjust the system's resonance frequency. As referred to [6], the efficiency can be easily derived from the equation (1).

$\eta_{12}=\frac{k_{T R}^{2} Q_{1} Q_{2 L}}{1+k_{T R}^{2} Q_{1} Q_{2 L}} \frac{Q_{2 L}}{Q_{L}}$

The parameter $k_{T R}$ represents the mutual magnetic coupling coefficient between both coils.

$k_{T R}=\frac{M_{T X-R X}}{\sqrt{L_{1} L_{2}}}$

While the theoretical (standalone) quality factors $Q_{1}, Q_{2}$ are both given by

$Q_{1,2}=\frac{\omega L_{1,2}}{R_{1,2}}$

in the real structure there is a significant influence of the mutual inductance between neighboring coils which measurably complicates its calculation [8] and [9]. The suitable computing method is described in the subsequent section. The parameter

$Q_{L}=\frac{R_{L}}{\omega L_{2}}$

from the equation (1) is often referred to as the load quality factor which in interaction with the $Q_{2}$ defines operational quality factor

\footnotetext{
* Vladimir Kindl, Tomas Kavalir, Roman Pechanek

Faculty of Electrical Engineering, University of West Bohemia, Plzen, Czech Republic

E-mail: vkindl@kev.zcu.cz
} 
of the last systems coil connected to the loading resistor $R_{L}$. The parameter

$Q_{2 L}=\frac{Q_{2} Q_{L}}{Q_{2}+Q_{L}}$

is the quality factor of the receiving circuit with load. From the foregoing thoughts it is clear that the large $Q_{1,2}$ and $k_{T R}$ positively affect (increase) the efficiency of the power transmission through the resonant magnetic coupling system.

\section{Key electrical parameters}

\section{A. Standalone Quality Factor}

The equation (3) describing the parameter was mentioned above. The angular frequency $\omega$ is set according to ISM band and therefore cannot be changed. The resistance and inductance are both strongly defined by the chosen geometry. While the planar square-shaped coil brings very high inductance and many construction advantages, it also exhibits considerable lateral parasitic capacity degrading the $Q$. This effect may be reduced by equidistantly wound coil turns, but on the other hand, it contrarily decreases back the inductance. With regard to previous statements the optimal shape of coils comes from all technical and geometrical requirements to be met. The exact value of selfinductance may be given either by FEA or from Grover's formula [10], [11] and [12] using the elliptical integrals.

\section{B. Operating Quality Factor}

If another coil appears within reach, the mutual inductance notably changes the numerator of (3). Moreover, there are several additional embedded resistances (see Fig. 2) having a significant reducing effect on the operational quality factor, especially the internal resistance of the power source $R_{G}$ and the load resistance $R_{L}$ of receiving resonant element. The situation may be easily explained by using the analogy of parallel resonant circuit. Both resonators $L_{1}-R_{1}-C_{T}$ and $L_{3}-R_{3}-C_{R}$ can be substitutely expressed by the parallel connection with permuted internal resistances of coils (see Fig. 3)

$R_{1 P}=\frac{L_{1}}{C_{T} R_{1}}$ and $R_{2 P}=\frac{L_{2}}{C_{R} R_{2}}$.

This new resonant circuit is further loaded with mentioned $R_{G}$ or $R_{L}$ linked in via matching capacitors (Fig. 2).

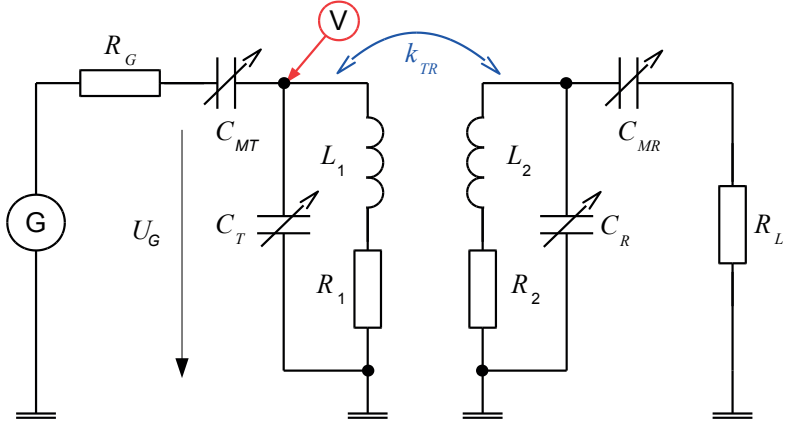

Fig. 2 The PSpice model of system's construction

As the loading impedance is connected in parallel to the resonator, we get the operating quality factor in following form

$Q_{p T}=\omega C_{T} \frac{R_{1 P} R_{G}}{R_{1 P}+R_{G}}$

and

$Q_{p R}=\omega C_{R} \frac{R_{2 P} R_{L}}{R_{2 P}+R_{L}}$

The substitutive parallel circuit is depicted in Fig. 3.

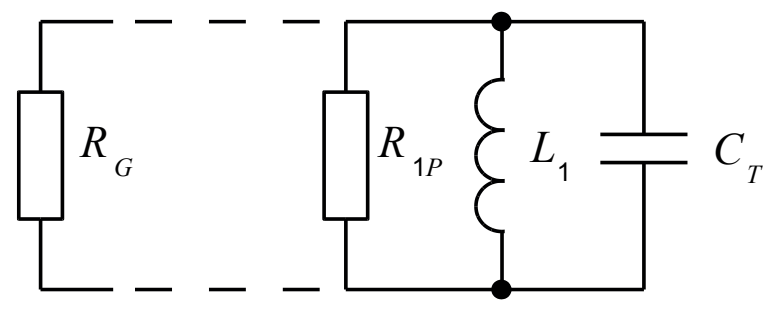

Fig. 3 The substitutive parallel resonant circuit-transceiver part

This budget of interlaced influences cannot be easily covered by simple analytical equation and must be therefore considered in another way [8] and [9]. To calculate the operational $Q$ correctly, we can assemble a simple PSpice model (see Fig. 2). The model performs an AC sweep analysis in which computes all necessary electrical quantities as they depend on the set frequency range. The quality factor can be further derived from the voltage curve measured on the resonator (see example in Fig. 4).

$Q_{R E S}=\frac{f_{0}}{B}=\frac{f_{0}}{f_{2}-f_{1}}$

The frequency $f_{0}$ matches the resonant frequency found in the extremum of the function from Fig. 3. The $f_{2}-f_{1}$ is the half-power bandwidth, i.e., the bandwidth over which the voltage is greater than $-3 \mathrm{~dB}$ of the voltage at the resonant frequency. 


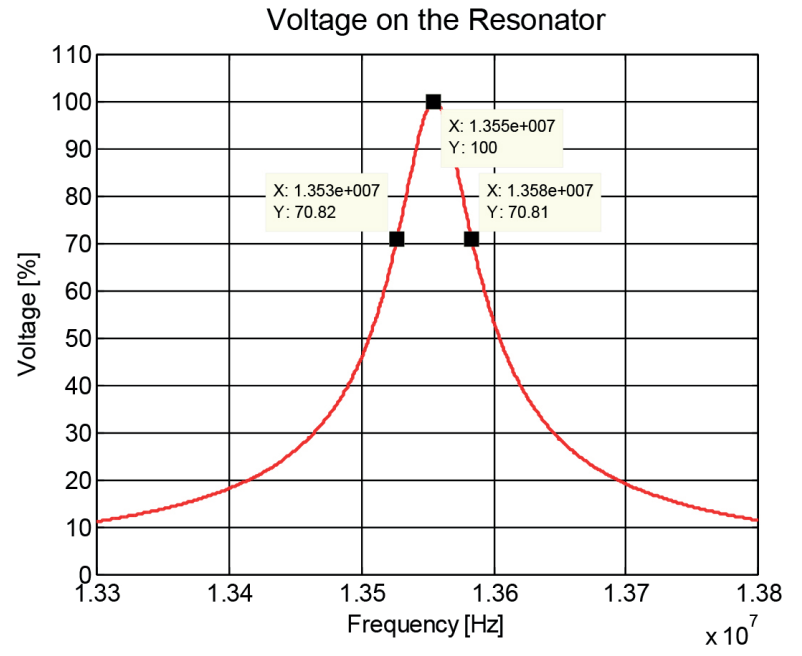

Fig. 4 Example for $Q$ calculation

Since the calculation combines influences of all components in the circuit, the following key parameters must be properly set into (1).

$Q_{1}=Q_{2}=Q_{R E S}$

\section{Structural Variations}

Discussed system is tested in three various structures composed of a combination of two types of planar coils (see Figs. 5 and 6).

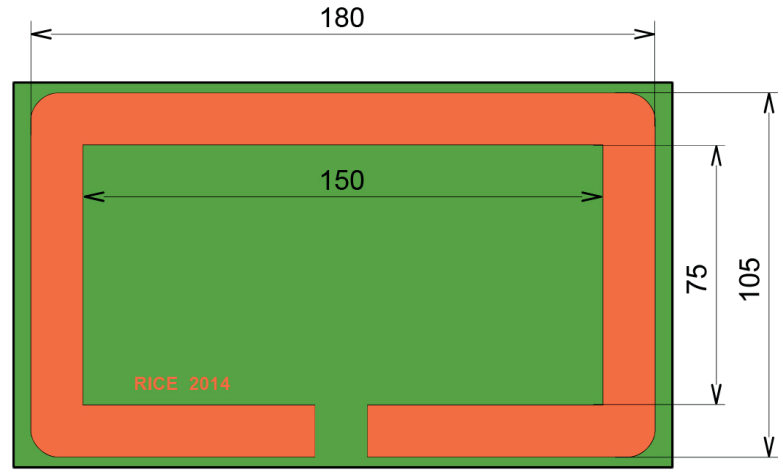

Fig. 5 The chart of planar coil - "version A"

While the coils are made as a pattern (PCB) on composite board FR4 or better [13], their manufacturing is very cheap, easy and quick. On the other hand, the lack of available space forbids linking the input power to the coil using magnetic coupling (capacitive coupling utilized). This negatively affects the ability to reach very high efficiency of power transmission. As compared with the coil "version B" (Fig. 6), the coil "version A" (Fig. 5) has higher effective area with only one turn to achieve higher quality factor with less sensitivity on near vicinity subjects.

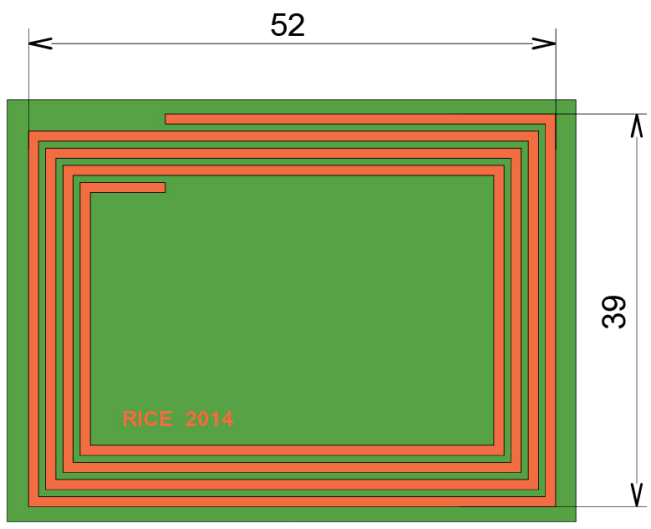

Fig. 6 The chart of planar coil - "version B"

The coil "version B" is made as a four-turn pattern of one millimeter thickness. The distance between each turn is $0.7 \mathrm{~mm}$. Since the coil is smaller and has lower quality factor, it finds some advantages in wider feasibility of mounting on moving parts. All important electrical parameters are listed in Table 1.

Important Electrical Parameters

Table 1

\begin{tabular}{|l|l|l|l|}
\hline & Parameter & Values & Units \\
\hline & $U_{G} / R_{G} / R_{L}$ & $10 / 50 / 50$ & {$[\mathrm{~V}] /[\Omega] /[\Omega]$} \\
\hline "version A" & $L_{1}$ & $278 \mathrm{e}^{-9}$ & {$[\mathrm{H}]$} \\
\hline "version A" & $C_{M T} / C_{T}$ & $23 \mathrm{e}^{-12} / 453 \mathrm{e}^{-12}$ & {$[\mathrm{~F}] /[\mathrm{F}]$} \\
\hline "version B" & $L_{2}$ & $1.3 \mathrm{e}^{-6}$ & {$[\mathrm{H}]$} \\
\hline "version B" & $C_{M R} / C_{R}$ & $13 \mathrm{e}^{-12} / 92 \mathrm{e}^{-12}$ & {$[\mathrm{~F}] /[\mathrm{F}]$} \\
\hline
\end{tabular}

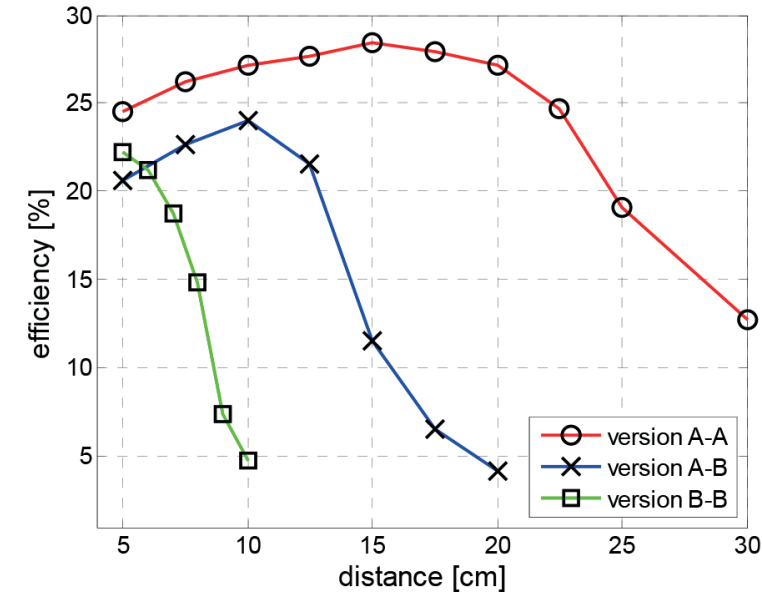

Fig. 7 The efficiency of WPT - all construction versions 
The first investigated framework utilizes two coils of "version A" for transmitting and receiving the power. Due to the same dimensions of both coils and their coaxial position during the measurement they exhibit relatively good efficiency even at very high distance. The resulting plot (red curve) for fixed frequency can be seen in Fig. 7.

The second tested configuration comprises the coil "version A" working as a transmitter and coil "version B" working as a receiver. Their unmatched dimensions decrease the maximum of reachable efficiency and short effective distance range. The resulting efficiency is depicted by a blue curve seen in Fig. 7 .

The third analyzed configuration consists only of the coils "version B". While the smaller coil features considerably lower quality factor than exhibited by the bigger one, the resulting efficiency and distance range must be according to (1) measurably lower. The resulting efficiency (green curve) is plotted in Fig. 7.

As it is clear from Fig. 7, the system exhibits at least one optimal distance at which the efficiency is maximal. This distance varies with the coils configuration whereas the higher $Q$ extends it. The starting low efficiency (small distance) is caused by increase of mutual inductance which consequently re-tunes the resonant frequency. This may be avoided either by using frequency tracking or tuning the resonant capacitors. Unfortunately, both countermeasures are either much expensive for real application, or may be implemented only with notable difficulties. The decrease in efficiency at large distances is mainly caused by the native character of near magnetic field (evanescent wave). According to (7) the lower impedance of source/load causes considerable decrease in the operating quality factor which makes the systems efficiency fall. Another important component of the system is the resonant capacitor itself. The quality of dielectric has strong impact on its internal resistance which is obviously parallel to the resonator and has therefore similar influence on the quality factor as well as the resistances from (6). For the practical application it is useful to pay attention even to the type dielectric of capacitors (low loss and low ESR).

The proposed system should be used to supply any measuring device for monitoring motor shaft parameters. As obvious, the smaller coil "version B" is more suitable for mounting on the shaft than the bigger coil "version A" and may be easily assembled in bond of many parallel coils connected to mutual DC bus (see Fig. 8).

If flexible PCB used, it is easy to cut this resulting elastic belt onto certain length and wound it on the shaft very quickly. The belt should be glued on strip of soft material (foam rubber) to get some distance from the shaft and to smooth its asperity. On the other hand, the smaller coil delivers the energy to the load with lower efficiency which produces stronger leakage flux having no negative impact on biological structures [14] in case of transferring low level energy. As a transmitter it is better to use the coil "version A" because of its less sensitivity on near objects and higher quality factor. The situation can be seen in Fig. 9.

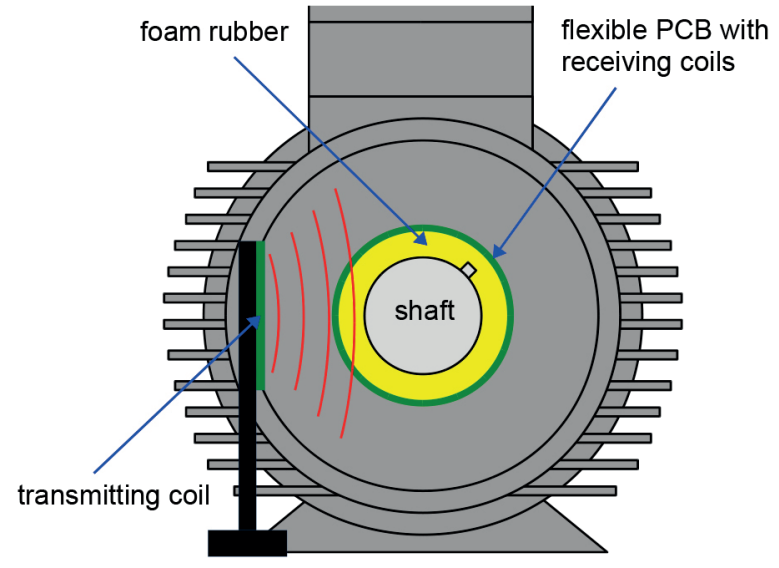

Fig. 9 System for WPT on the shaft

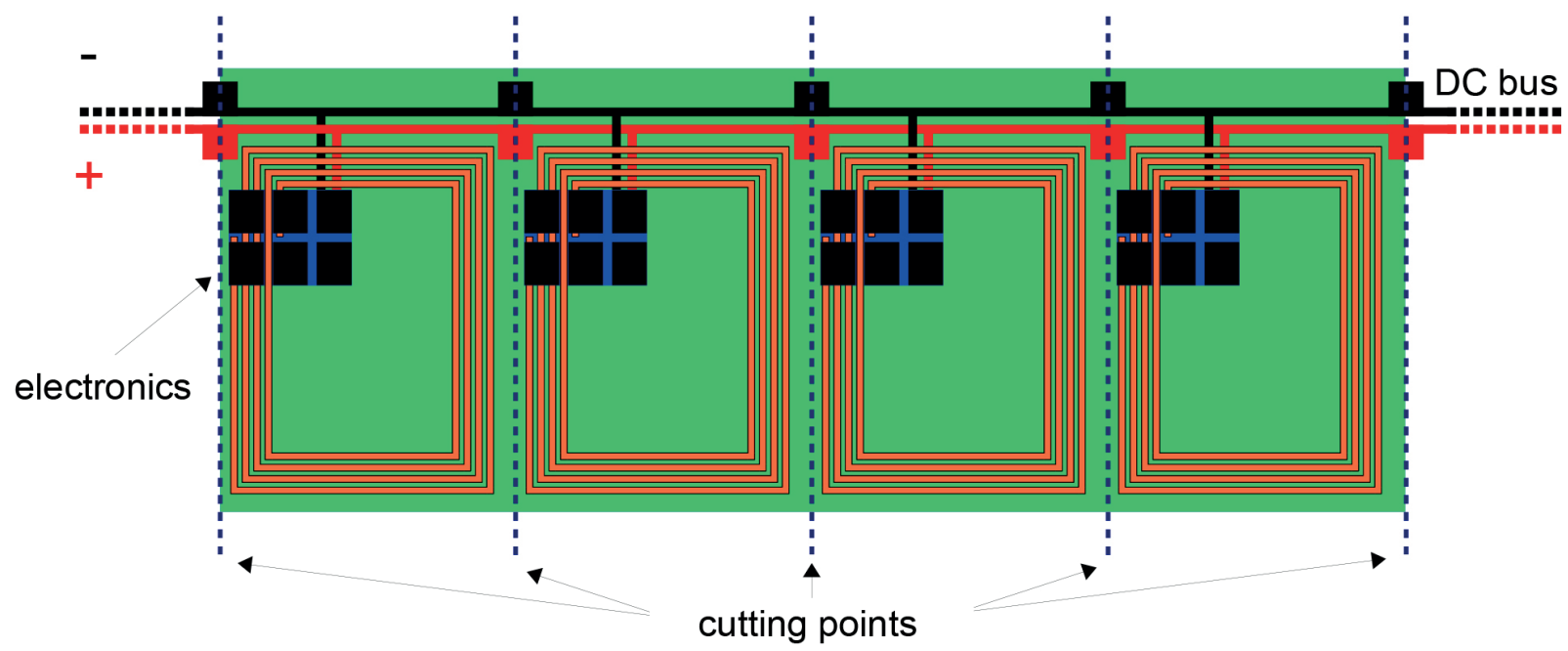

Fig. 8 Flexible PCB to be mounted on the shaft 


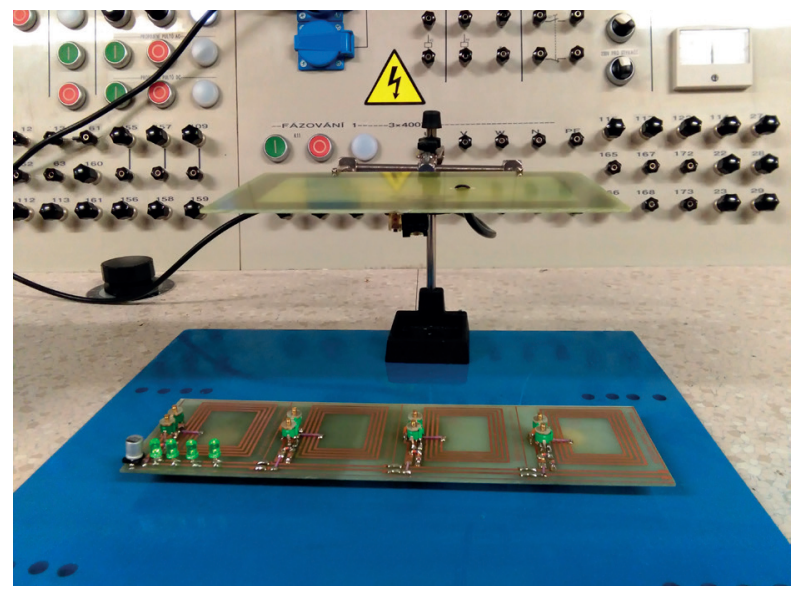

Fig. 10 The real shape of tested arrangement - demonstration

The real shape of tested (demonstrating) system is shown in Fig. 10. The receiving coils are placed on metal plate with $10 \mathrm{~mm}$ distance.

\section{Laboratory arrangement for measuring}

The measuring arrangement can be seen in Fig. 11. Here the radio transceiver FT-857D is used as a source of transmitting energy. The operational frequency is $13.56 \mathrm{MHz}$, which is permitted by the ISM band. The input/output power is measured by the Watt-meter $\mathrm{CN}-801$ (type $\mathrm{H}$ ) of $200 \mathrm{MHz}$ bandwidth and relatively large power range $(20 / 200 / 2000 \mathrm{~W})$. The impedance was matched by linking capacitors $C_{M T}$ and $C_{M R}$.

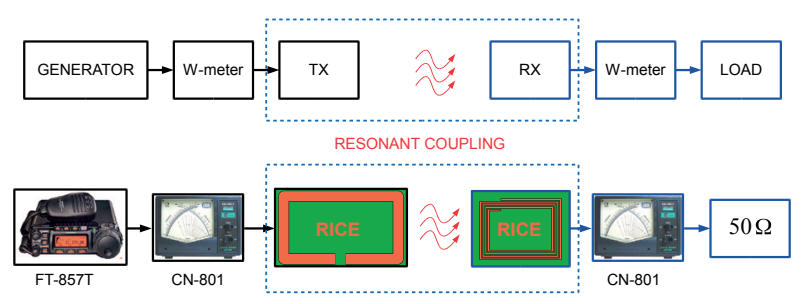

Fig. 11 The measuring arrangement

\section{Conclusions}

The experimental results indicate relatively high sensitivity on making alternations to the system arrangement. From the outcome of our investigation it is possible to give several conclusions.

The first, the system exhibits at least one optimal coils' distance in which the efficiency is maximal. This is true only for the constant supplying frequency and it is therefore possible to partially improve the short-distance efficiency. The improvement may be done either by using frequency tracking or tuning the resonant capacitors. The decrease in efficiency at large distances is mainly caused by the native character of near magnetic field (evanescent wave). The magnetic field range can be effectively extended by increasing in coils' quality factor.

The second, the full-range efficiency of the system is also strongly influenced by the internal impedance of the power source and the load itself. Another key parameter is the quality of dielectric of the resonant capacitor. It has strong impact on its internal resistance which is obviously parallel to the resonator and has therefore the similar influence on the quality factor as exhibited by source/load resistance. The findings suggest that despite of the higher cost it is better to choose the low loss and low ESR capacitors making the system efficient even at large distances.

The proposed receiving coil "version B" is small enough to be easily assembled in bond of many repeating parallel coils having the belt-shape. All coils may be connected to mutual DC bus which together with flexible PCB makes possibility to cut the structure at any required length and mount it on the shaft very quickly. The foam rubber strip glued on the bottom side of the belt makes some distance between the shaft and mounted coils and smoothes any asperity of the surface. While the dimensions of transmitting coil are not so limited, it is better to use the (larger) coil with higher $Q$ to achieve less sensitivity on precise mutual position and near disturbing objects.

\section{Acknowledgements}

This research has been supported by the European Regional Development Fund and the Ministry of Education, Youth and Sports of the Czech Republic under the Regional Innovation Centre for Electrical Engineering (RICE), project No. CZ.1.05/2.1.00/03.0094, TACR project No. TA02000103 CIDAM and funding program SGS-2015-038.

\section{References}

[1] SANGCHEOL, M., BONG-CHUL, K., SHIN-YOUNG, CH., GUN-WOO, M.: Analysis and Design of Wireless Power Transfer System with an Intermediate Coil for High Efficiency, ECCE Asia Downunder (ECCE Asia), 2013 IEEE , pp.1034-1040, June 2013

[2] HEE-SEUNG, K., DO-HYUN, W., BYUNG-JUN, J.: Simple Design Method of Wireless Power Transfer System Using 13.56MHz Loop Antennas, Industrial Electronics (ISIE), 2010 IEEE Intern. Symposium, pp. 1058-1063, July 2010 
[3] ZHEN NING, L., CHINGA, R. A., RYAN, T., JENSHAN, L.: Design and Test of a High-Power High-Efficiency Loosely Coupled Planar Wireless Power Transfer System, Industrial Electronics, IEEE Transactions, vol. 56, No. 5, pp.1801-1812, May 2009

[4] YIMING, Z., ZHENGMING, Z.: Frequency Splitting Analysis of Two-Coil Resonant Wireless Power Transfer, Antennas and Wireless Propagation Letters, IEEE, vol.13, No., pp. 400-402, 2014

[5] ZHANG, Y.; LU, T.; ZHAO, Z.; HE, F.; CHEN, K.; YUAN, L.: Selective Wireless Power Transfer to Multiple Loads Using Receivers of Different Resonant Frequencies, Power Electronics, IEEE Transactions, No. 99.

[6] KIANI, M., GHOVANLOO, M.: The Circuit Theory Behind Coupled-Mode Magnetic Resonance-Based Wireless Power Transmission, Circuits and Systems I, Regular Papers, IEEE Transactions, vol. 59, No. 9, pp. 2065-2074

[7] KUSAKA, K.; ITOH, J.-I.: Experimental Verification of Rectifiers with SiC/GaN for Wireless Power Transfer Using a Magnetic Resonance Coupling, Power Electronics and Drive Systems (PEDS), IEEE Ninth Intern. Conference, pp.1094-1099, Dec. 2011

[8] TOOLEY, M, TOOLEY, M. H.: Electronic Circuits: Fundamentals and Applications, Newnes, pp. 77-78, ISBN 978-0-7506-6923-8

[9] CHUNLI, W, ZHIMING, B, JINGKUI, X, JINXING, W.: Simulation Analysis on Quality Factor of RF Receiving Coil for an MRI System, Control and Decision Conference, CCDC '09, Chinese, pp. 4652-4655, June 2009

[10] BABIC, S. I., SIROIS, F., AKYEL, C.; Validity Check of Mutual Inductance Formulas for Circular Filaments With Lateral and Angular Misalignments, Progress in Electromagnetics Research M, vol. 8, pp. 15-26, 2009

[11] BABIC, S. I., AKYEL, C.: Calculating Mutual Inductance between Circular Coils with Inclined Axes in Air, Magnetics, IEEE Transactions, vol. 44, No. 7, pp. 1743-1750, July 2008

[12] BABIC, S., AKYEL, C.: Improvement in Calculation of the Self- and Mutual Inductance of Thin-wall Solenoids and Disk Coils, Magnetics, IEEE Transactions, vol. 36, No. 4, pp. 1970-1975, July 2000

[13] HOCKICKO, P., BURY, P., MUNOZ, F.: Analyses of Dielectric Properties of Lithium Phosphate Glasses, Communications Scientific Letters of the University of Zilina, No. 2A, 2013

[14] RADIL, R., JANOUSEK. L.: Yeast Growth Influenced by Parallel Combination of Time-Varying and Static LF EMF, Communications - Scientific Letters of the University of Zilina, No. 2A, 2013. 